\title{
Large and moderate deviations of realized covolatility
}

\author{
Hacène Djellout*, Yacouba Samoura \\ Laboratoire de Mathématiques, CNRS UMR 6620, Université Blaise Pascal, Avenue des Landais, BP80026, 63171 Aubière Cedex, France
}

\section{A R T I C L E I N F O}

\section{Article history:}

Received 17 June 2013

Received in revised form 3 December 2013

Accepted 3 December 2013

Available online 25 December 2013

\section{MSC:}

$60 \mathrm{~F} 10$

$62 \mathrm{~J} 05$

$60 \mathrm{~J} 05$

\section{Keywords:}

Deviation inequalities

Large and moderate deviation principle

Diffusion

Discrete-time observation

Realized volatility

\begin{abstract}
A B S T R A C T
In this note, we consider the large and moderate deviation principle of the estimators of the integrated covariance of two-dimensional diffusion processes when they are observed only at discrete times in a synchronous manner. The proof is extremely simple. It is essentially an application of the contraction principle for the results given in the case of the volatility by Djellout et al. (1999).
\end{abstract}

(C) 2014 Elsevier B.V. All rights reserved.

\section{Motivation and context}

Given a filtered probability space $\left(\Omega, \mathcal{F},\left(\mathcal{F}_{t}\right), \mathbb{P}\right)$, let $\left(X_{1, t}, X_{2, t}\right)$ be a two-dimensional diffusion process given by

$$
\left\{\begin{array}{l}
d X_{1, t}=u_{1, t}\left(X_{1, t}\right) d t+\sigma_{1, t} d B_{1, t} \\
d X_{2, t}=u_{2, t}\left(X_{2, t}\right) d t+\sigma_{2, t} d B_{2, t}
\end{array}\right.
$$

where $\left(\left(B_{1, t}, B_{2, t}\right), t \geq 0\right)$ is a two-dimensional Gaussian process with independent increments, zero mean and covariance matrix

$$
\left(\begin{array}{cc}
t & \int_{0}^{t} \rho_{s} d s \\
\int_{0}^{t} \rho_{s} d s & t
\end{array}\right) \forall t \geq 0 .
$$

In (1.1), $\left(u_{1}, u_{2}\right)$ is a progressively measurable process (possibly unknown). In what follows, we restrict our attention to the case when $\sigma_{1}, \sigma_{2}$ and $\rho$ are deterministic functions; the functions $\sigma_{i}, i=1,2$ take positive values while $\rho$ takes values in the interval $[-1,1]$. Note that the marginal processes $B_{1}$ and $B_{2}$ are Brownian motions (BM). Moreover, we can define a process $B_{t}^{*}$ such that $\left(B_{1, t}, B_{t}^{*}\right)_{t \geq 0}$ is a two-dimensional BM and $d B_{2, t}=\rho_{t} d B_{1, t}+\sqrt{1-\rho_{t}^{2}} d B_{t}^{*}$ for every $t \geq 0$.

\footnotetext{
* Corresponding author. Fax: +33473407064.

E-mail addresses: Hacene.Djellout@math.univ-bpclermont.fr (H. Djellout), Samoura.Yacouba@math.univ-bpclermont.fr (Y. Samoura).
} 
In this note, the parameter of interest is the (deterministic) covariance of $X_{1}$ and $X_{2}$

$$
\left\langle X_{1}, X_{2}\right\rangle_{t}=\int_{0}^{t} \sigma_{1, t} \sigma_{2, t} \rho_{t} d t .
$$

In finance, $\left\langle X_{1}, X_{2}\right\rangle$. is the integrated covariance (over [0,1]) of the logarithmic prices $X_{1}$ and $X_{2}$ of two securities. It is an essential quantity to be measured for risk management purposes. The covariance for multiple price processes is of great interest in many financial applications. The naive estimator is the realized covariance, which is the analogue of realized variance for a single process.

Typically $X_{1, t}$ and $X_{2, t}$ are not observed in continuous time but we have only discrete time observations. Given discrete equally spaced observation $\left(X_{1, t_{k}^{n}}, X_{2, t_{k}^{n}}, k=1, \ldots, n\right)$ in the interval $[0,1]$ (with $t_{k}=k / n$ ), a commonly used approach to estimate is to take the sum of cross products

$$
\mathbf{C}_{t}^{n}:=\sum_{k=1}^{[n t]}\left(X_{1, t_{k}^{n}}-X_{1, t_{k-1}^{n}}\right)\left(X_{2, t_{k}^{n}}-X_{2, t_{k-1}^{n}}\right),
$$

where $[x]$ denotes the integer part of $x \in \mathbb{R}$.

When the drift is known, we can also consider the following estimator:

$$
\overline{\mathbf{C}}_{t}^{n}:=\sum_{k=1}^{[n t]}\left(X_{1, t_{k}^{n}}-X_{1, t_{k-1}^{n}}-\int_{t_{k-1}^{n}}^{t_{k}^{n}} u_{1, t}\left(X_{1, t}\right) d t\right)\left(X_{2, t_{k}^{n}}-X_{2, t_{k-1}^{n}}-\int_{t_{k-1}^{n}}^{t_{k}^{n}} u_{2, t}\left(X_{2, t}\right) d t\right) .
$$

In the unidimensional case and in the case that $X$ have non-jump, this question has been well investigated-see Djellout et al. (1999) for relevant references. In Djellout et al. (1999) and recently in Kanaya and Otsu (2012), the authors obtained the large and moderate deviations for the realized volatility. The results of Djellout et al. (1999) are extended to jump-diffusion processes. Mancini (2008) established the large deviation result for the threshold estimator for the constant volatility. Jiang (2010) derived a moderate deviation result for the threshold estimator for the quadratic variational process.

In the bivariate case, Hayashi and Yoshida (2011) considered the problem of estimating the covariation of two diffusion processes under a non-synchronous sampling scheme. They proposed an alternative estimator and they investigated the asymptotic distributions. In Dalalyan and Yoshida (2011), the authors complement the results in Hayashi and Yoshida (2011) by establishing a second-order asymptotic expansion for the distribution of the estimator in a fairly general setup, including random sampling schemes and (possibly random) drift terms. Several further works have been realized when data on two securities are observed non-synchronously, see also Aït-Sahalia et al. (2010). Here we do not consider the asynchronous case. In the bivariate case we also mention the work of Mancini and Gobbi (2012) which deals with the problem of distinguishing the Brownian covariation from the co-jumps using a discrete set of observations.

The purpose of this note is to furnish some further estimations about the estimator (1.3), refining the already known central limit theorem. More precisely, we are interested in the estimations of

$$
\mathbb{P}\left(\frac{\sqrt{n}}{b_{n}}\left(\mathbf{C}_{t}^{n}-\int_{0}^{t} \sigma_{1, t} \sigma_{2, t} \rho_{t} d t\right) \in A\right),
$$

where $A$ is a given domain of deviation, and $\left(b_{n}\right)_{n>0}$ is some sequence denoting the scale of the deviation. When $b_{n}=1$, this is exactly the estimation of the central limit theorem. When $b_{n}=\sqrt{n}$, it becomes the large deviations. And when $1 \ll b_{n} \ll \sqrt{n}$, this is the so-called moderate deviations. The main problem studied in this paper is the large and moderate deviation estimations of the estimator. In this bivariate case things are not complicated.

We refer to Dembo and Zeitouni (1998) for an exposition of the general theory of large deviation and limit ourself to the statement of the some basic definitions. Let $\left\{\mu_{T}, T>0\right\}$ be a family of probability on a topological space $(S, \delta)$ where $s$ is a $\sigma$-algebra on $S$ and $v(T)$ a non-negative function on $[1, \infty)$, such that $\lim _{T \rightarrow \infty} v(T)=+\infty$. A function $I: S \rightarrow[0, \infty]$ is said to be a rate function if it is lower semicontinuous and it is said to be a good rate function if its level set $\{x \in S: I(x) \leq a\}$ is compact for all $a \geq 0 .\left\{\mu_{T}\right\}$ is said to satisfy a large deviation principle (LDP) with speed $v(T)$ and rate function $I(x)$ if for any set $A \in \&$

$$
-\inf _{x \in A^{\circ}} I(x) \leq \lim _{T \rightarrow \infty}\left(\begin{array}{l}
\inf \\
\sup
\end{array}\right) \frac{1}{v(T)} \log \mu_{T}(A) \leq-\inf _{x \in \bar{A}} I(x),
$$

where $A^{0}, \bar{A}$ are the interior and the closure of $A$ respectively.

This paper is organized as follows. In the next section we present the main results of this paper. They are established in the last section.

\section{Main results}

Our first result is about the $\operatorname{LDP}$ of $\mathbb{P}\left(\mathbf{C}_{1}^{n} \in \cdot\right)$, with time $t=1$ fixed. 
Proposition 2.1. Let $\left(X_{1, t}, X_{2, t}\right)$ be given by (1.1).

(1) For every $\lambda \in \mathbb{R}$

$$
\begin{aligned}
\Lambda_{n}(\lambda) & :=\frac{1}{n} \log \mathbb{E}\left(\exp \left(\lambda n \overline{\mathbf{C}}_{1}^{n}\right)\right) \\
& \leq \Lambda(\lambda):=\left\{\begin{array}{l}
\int_{0}^{1}-\frac{1}{2} \log \left(1-\lambda \sigma_{1, t} \sigma_{2, t}\left(1+\rho_{t}\right)\right)-\frac{1}{2} \log \left(1+\lambda \sigma_{1, t} \sigma_{2, t}\left(1-\rho_{t}\right)\right) d t \\
\text { if }-\frac{1}{\left\|\sigma_{1} \sigma_{2}(1-\rho)\right\|} \leq \lambda \leq \frac{1}{\left\|\sigma_{1} \sigma_{2}(1+\rho)\right\|} \\
+\infty, \quad \text { otherwise }
\end{array}\right.
\end{aligned}
$$

and

$$
\lim _{n \rightarrow \infty} \Lambda_{n}(\lambda)=\Lambda(\lambda)
$$

(2) Assume that $\sigma_{1,}, \sigma_{2, .}(1 \pm \rho.) \in L^{\infty}([0,1], d t)$ and $u_{l, \cdot}(\cdot) \in L^{\infty}(d t \otimes \mathbb{P})$, for $l=1$, 2. Then $\mathbb{P}\left(\mathbf{C}_{1}^{n} \in \cdot\right)$ satisfies the LDP on $\mathbb{R}$ with speed $n$ and with the good rate function given by the Legendre transformation of $\Lambda$, that is

$$
\Lambda^{*}(x)=\sup _{\lambda \in \mathbb{R}}\{\lambda x-\Lambda(\lambda)\} .
$$

We now extend Proposition 2.1 to the process-level large deviations of $\mathbb{P}\left(\mathbf{C}^{n} \in \cdot\right)$, which is interesting from the viewpoint of the non-parametric statistics.

Let $\mathbb{D}_{b}([0,1])$ be the real right-continuous-left-limit and bounded variation functions $\gamma$. The space $\mathbb{D}_{b}([0,1])$ of $\gamma$, identified in the usual way as the space of bounded measures $d \gamma$ on [0,1], with $d \gamma[0, t]=\gamma(t)$ and $d \gamma(0)=\gamma(0)$, will be equipped with the weak convergence topology and the $\sigma$-field $\mathscr{B}^{s}$ generated by the coordinate $\{\gamma(t), 0 \leq t \leq 1\}$. We denote by $\dot{\gamma}(t) d t$ and $d \gamma^{\perp}$ respectively the absolute continuous part and the singular part of the measure $d \gamma$ associated with $\gamma \in \mathbb{D}_{b}[0,1]$ w.r.t. the Lebesgue measure $d t$. The signed measure $\gamma$ has a unique decomposition into a difference $\gamma=\gamma_{+}-\gamma_{-}$of two positive measures $\gamma_{+}$and $\gamma_{-}$. In the paper, we denote by $P^{*}$ the function

$$
P^{*}(x)= \begin{cases}\frac{1}{2}(x-1-\log x) & \text { if } x>0 \\ +\infty & \text { if } x \leq 0\end{cases}
$$

which is the Legendre transformation of $P$ given by

$$
P(\lambda)= \begin{cases}-\frac{1}{2} \log (1-2 \lambda) & \text { if } \lambda<\frac{1}{2} \\ +\infty, & \text { otherwise. }\end{cases}
$$

Theorem 2.2. Let $\left(X_{1, t}, X_{2, t}\right)$ be given by (1.1). Assume that $\sigma_{1, \cdot} \sigma_{2, .}(1 \pm \rho.) \in L^{\infty}([0,1], d t)$ and $u_{l, \cdot}(\cdot)=u_{l}(\cdot, \cdot) \in L^{\infty}(d t \otimes \mathbb{P})$, for $l=1$, 2. Then

(1) $\mathbb{P}\left(\mathbf{C}^{n} \in \cdot\right)$ satisfies the $L D P$ on $\mathbb{D}_{b}([0,1])$ w.r.t. the weak convergence topology, with speed $n$ and with some inf-compact convex rate function $J(\gamma)$.

(2) If moreover $t \rightarrow \sigma_{1, t} \sigma_{2, t}\left(1 \pm \rho_{t}\right)$ is continuous and strictly positive on [0, 1], then

$$
J(\gamma)=J_{+}^{a b s}\left(\gamma_{+}+\beta\right)+J_{-}^{a b s}\left(\gamma_{-}+\beta\right)+J_{+}^{\perp}\left(\gamma_{+}\right)+J_{-}^{\perp}\left(\gamma_{-}\right),
$$

where $\beta$ is absolutely continuous with respect to the Lebesgue measure and given by

$$
\begin{aligned}
\dot{\beta}(t)= & \frac{\sigma_{1, t} \sigma_{2, t}\left(1-\rho_{t}^{2}\right)-\left(\dot{\gamma}_{+}(t)+\dot{\gamma}_{-}(t)\right)}{2} \\
& +\frac{\sqrt{\left[\sigma_{1, t} \sigma_{2, t}\left(1-\rho_{t}^{2}\right)-\left(\dot{\gamma}_{+}(t)+\dot{\gamma}_{-}(t)\right)\right]^{2}+\left(\dot{\gamma}_{+}(t)+\dot{\gamma}_{-}(t)\right) \sigma_{1, t} \sigma_{2, t}\left(1-\rho_{t}^{2}\right)}}{2},
\end{aligned}
$$

and

$$
J_{ \pm}^{\perp}(\gamma)=\int_{0}^{1} \frac{1}{\sigma_{1, t} \sigma_{2, t}\left(1 \pm \rho_{t}\right)} d \gamma^{\perp}
$$

and

$$
J_{ \pm}^{a b s}(\gamma)=\int_{0}^{1} P^{*}\left(\frac{2 \dot{\gamma}(t)}{\sigma_{1, t} \sigma_{2, t}\left(1 \pm \rho_{t}\right)}\right) d t,
$$

where $P^{*}$ is given in (2.2). 

that

We discuss now the moderate deviation principle. To this purpose, let $\left(b_{n}\right)_{n \geq 1}$ be a sequence of positive numbers such

$$
b_{n} \rightarrow \infty \text { and } \frac{b_{n}}{\sqrt{n}} \rightarrow 0 \text { as } n \rightarrow \infty .
$$

Let $\mathbb{D}_{0}[0,1]$ be the Banach space of real right-continuous-left-limit functions $\gamma$ on $[0,1]$ with $\gamma(0)=0$, equipped with the uniform sup norm and the $\sigma$-field $\mathscr{B}^{s}$ generated by the coordinate $\{\gamma(t), 0 \leq t \leq 1\}$.

Theorem 2.3. Given $\left(X_{1, t}, X_{2, t}\right)$ by $(1.1)$ with $u_{l, .}(\cdot)=u_{l}(\cdot, \cdot) \in L^{\infty}(d t \otimes \mathbb{P})$, for $l=1$, 2. Assume that $\sigma_{1, .} \sigma_{2, .}(1 \pm \rho.) \in$ $L^{2}([0,1], d t)$ and

$$
\sqrt{n} b_{n} \max _{1 \leq k \leq n} \int_{(k-1) / n}^{k / n} \sigma_{1, t} \sigma_{2, t}\left(1 \pm \rho_{t}\right) d t \longrightarrow 0 .
$$

Then $\mathbb{P}\left(\frac{\sqrt{n}}{b_{n}}\left(\mathbf{C}^{n}-\left\langle X_{1}, X_{2}\right\rangle.\right) \in \cdot\right)$ satisfies the $L D P$ on $\mathbb{D}_{0}([0,1])$ with speed $b_{n}^{2}$ and with the good rate function $J_{m}$ given by

$$
J_{m}(\gamma)=\left\{\begin{array}{l}
\int_{0}^{1} \frac{\dot{\gamma}(t)^{2}}{2 \sigma_{1, t}^{2} \sigma_{2, t}^{2}\left(1+\rho_{t}^{2}\right)} 1_{\left[t: \sigma_{1, t} \sigma_{2, t}>0\right]} d t \quad \text { if } d \gamma \ll \sigma_{1, t} \sigma_{2, t} \sqrt{1+\rho_{t}^{2}} d t \\
+\infty \text { otherwise. }
\end{array}\right.
$$

Remark 2.4. In particular, $\mathbb{P}\left(\frac{\sqrt{n}}{b_{n}}\left(\mathbf{C}_{1}^{n}-\left\langle X_{1}, X_{2}\right\rangle_{1}\right) \in \cdot\right)$ satisfies the LDP on $\mathbb{R}$ with speed $b_{n}^{2}$ and with the rate function given by

$$
I_{m}(x)=\frac{x^{2}}{2 \int_{0}^{1} \sigma_{1, s}^{2} \sigma_{2, s}^{2}\left(1+\rho_{s}^{2}\right) d s}, \quad \forall x \in \mathbb{R}
$$

Remark 2.5. If for some $p>2$,

$$
\sigma_{1,} \sigma_{2, .}(1 \pm \rho .) \in L^{p}([0,1], d t) \quad \text { and } \quad b_{n}=O\left(n^{\frac{1}{2}-\frac{1}{p}}\right),
$$

we obtain (2.5).

Remark 2.6. Theorems 2.2 and 2.3 continue to hold under the linear growth condition of the drift $u_{l}(l=1,2)$ rather than the boundedness. More precisely assume that

$$
\left|u_{l, s}(x)-u_{l, t}(y)\right| \leq \alpha_{l}\left[1+|x-y|+\eta_{l}(|s-t|)(|x|+|y|)\right], \quad \forall s, t \in[0,1], x, y \in \mathbb{R},
$$

where $\eta_{l}:[0,+\infty) \rightarrow[0,+\infty)$ is a continuous nondecreasing function with $\eta_{l}(0)=0$ and $\alpha_{l}>0$ is a constant. Then the LDP of Theorems 2.2 and 2.3 continue to hold for $\mathbb{P}\left(\tilde{\mathbf{C}}^{n} \in \cdot\right)$, where $\tilde{\mathbf{C}}^{n}$ is given by

$$
\tilde{\mathbf{C}}_{t}^{n}:=\sum_{k=1}^{[n t]}\left(X_{1, t_{k}^{n}}-X_{1, t_{k-1}^{n}}-u_{1, t_{k-1}^{n}}\left(X_{1, t_{k-1}^{n}}\right)\left(t_{k}^{n}-t_{k-1}^{n}\right)\right)\left(X_{2, t_{k}^{n}}-X_{2, t_{k-1}^{n}}-u_{2, t_{k-1}^{n}}\left(X_{2, t_{k-1}^{n}}\right)\left(t_{k}^{n}-t_{k-1}^{n}\right)\right) .
$$

We introduce the following function:

$$
\Lambda_{ \pm}^{*}(x)=\sup _{\lambda \in \mathbb{R}}\left\{\lambda x-\Lambda_{ \pm}(\lambda)\right\}
$$

which is the Legendre transformation of $\Lambda_{ \pm}$given by

$$
\Lambda_{ \pm}(\lambda):=\int_{0}^{1} P\left( \pm \frac{\lambda \sigma_{1, t} \sigma_{2, t}\left(1 \pm \rho_{t}\right)}{2}\right) d t
$$

and we denote

$$
\alpha_{ \pm, t}=\frac{1}{2} \int_{0}^{t} \sigma_{1, s} \sigma_{2, s}\left(1 \pm \rho_{s}\right) d s
$$

An easy application of deviation inequalities given in Proposition 1.5 in Djellout et al. (1999) gives the following proposition. 
Proposition 2.7. We have for every $n \geq 1$ and $r>0$,

$$
\begin{aligned}
\mathbb{P}\left(\sup _{t \in[0,1]}\left[\overline{\mathbf{C}}_{t}^{n}-\mathbb{E} \overline{\mathbf{C}}_{t}^{n}\right] \geq r\right) \leq & \exp \left(-n \Lambda_{+}^{*}\left(\alpha_{+}+\frac{r}{2}\right)\right)+\exp \left(-n \Lambda_{-}^{*}\left(\alpha_{-}-\frac{r}{2}\right)\right) \\
\leq & \exp \left(-\frac{n}{2}\left[\frac{r}{\left\|\sigma_{1} \sigma_{2}(1+\rho)\right\|_{\infty}}-\log \left(1+\frac{r}{\left\|\sigma_{1} \sigma_{2}(1+\rho)\right\|_{\infty}}\right)\right]\right) \\
& +\exp \left(-n \frac{r^{2}}{4 \int_{0}^{1}\left[\sigma_{1} \sigma_{2}(1-\rho)\right]^{2} d t}\right), \\
\mathbb{P}\left(\inf _{t \in[0,1]}\left[\overline{\mathbf{C}}_{t}^{n}-\overline{\mathbf{C}}_{t}^{n}\right] \leq-r\right) \leq & \exp \left(-n \Lambda_{+}^{*}\left(\alpha_{+}-\frac{r}{2}\right)\right)+\exp \left(-n \Lambda_{-}^{*}\left(\alpha_{-}+\frac{r}{2}\right)\right) \\
\leq & \exp \left(-n \frac{r^{2}}{4 \int_{0}^{1}\left[\sigma_{1} \sigma_{2}(1+\rho)\right]^{2} d t}\right) \\
& +\exp \left(-\frac{n}{2}\left[\frac{r}{\left\|\sigma_{1} \sigma_{2}(1-\rho)\right\|_{\infty}}-\log \left(1+\frac{r}{\left\|\sigma_{1} \sigma_{2}(1-\rho)\right\|_{\infty}}\right)\right]\right),
\end{aligned}
$$

where $\Lambda_{ \pm}^{*}$ and $\alpha_{ \pm}$are given in (2.7) and (2.9) respectively.

\section{Proof}

In this section, we will give some hints for the proof of the main results. We have

$$
\overline{\mathbf{C}}_{t}^{n}=\sum_{k=1}^{[n t]}\left(\int_{t_{k-1}^{n}}^{t_{k}^{n}} \sigma_{1, s} d B_{1, s}\right)\left(\int_{t_{k-1}^{n}}^{t_{k}^{n}} \sigma_{2, s} d B_{2, s}\right)=\sum_{k=1}^{[n t]} \sqrt{a_{k}} \sqrt{a_{k}^{\prime}} \xi_{k} \xi_{k}^{\prime},
$$

where

$$
\xi_{k}=\frac{\int_{t_{k-1}^{n}}^{t_{k}^{n}} \sigma_{1, s} d B_{1, s}}{\sqrt{a_{k}}} \quad \xi_{k}^{\prime}=\frac{\int_{t_{k-1}^{n}}^{t_{k}^{n}} \sigma_{2, s} d B_{2, s}}{\sqrt{a_{k}^{\prime}}} \quad \text { with } a_{k}=\int_{t_{k-1}^{n}}^{t_{k}^{n}} \sigma_{1, t}^{2} d t a_{k}^{\prime}=\int_{t_{k-1}^{n}}^{t_{k}^{n}} \sigma_{2, t}^{2} d t .
$$

Obviously $\left(\left(\xi_{k}, \xi_{k}^{\prime}\right)\right)_{k=1, \ldots, n}$ are independent centered Gaussian random vector with covariance

$$
\frac{1}{\sqrt{a_{k}} \sqrt{a_{k}^{\prime}}}\left(\begin{array}{cc}
\sqrt{a_{k}} \sqrt{a_{k}^{\prime}} & \int_{t_{k-1}^{n}}^{t_{k}^{n}} \sigma_{1, s} \sigma_{2, s} \rho_{s} d s \\
\int_{t_{k-1}^{n}}^{t_{k}^{n}} \sigma_{1, s} \sigma_{2, s} \rho_{s} d s & \sqrt{a_{k}} \sqrt{a_{k}^{\prime}}
\end{array}\right) .
$$

Let us introduce the following notation:

$$
\mathbf{Q}_{ \pm, t}^{n}=\frac{1}{4} \sum_{k=1}^{[n t]} \sqrt{a_{k}} \sqrt{a_{k}^{\prime}}\left(\xi_{k} \pm \xi_{k}^{\prime}\right)^{2} .
$$

The proof relies on the following decomposition:

$$
\overline{\mathbf{C}}_{t}^{n}=\mathbf{Q}_{+, t}^{n}-\mathbf{Q}_{, t}^{n} \text {. }
$$

Proof of Proposition 2.1. By the independence of $\mathbf{Q}_{+, 1}^{n}$ and $\mathbf{Q}^{n}{ }_{, 1}$, we obtain that

$$
\begin{aligned}
\Lambda_{n}(\lambda) & =\frac{1}{n} \log \mathbb{E}\left(\exp \left(\lambda n \overline{\mathbf{C}}_{1}^{n}\right)\right)=\frac{1}{n} \log \mathbb{E}\left(\exp \left(\lambda n\left(\mathbf{Q}_{+, 1}^{n}-\mathbf{Q}_{,, 1}^{n}\right)\right)\right) \\
& =\frac{1}{n} \log \mathbb{E}\left(\exp \left(\lambda n \mathbf{Q}_{+, 1}^{n}\right)\right)+\frac{1}{n} \log \mathbb{E}\left(\exp \left(-\lambda n \mathbf{Q}_{,, 1}^{n}\right)\right):=\Lambda_{n,+}(\lambda)+\Lambda_{n,-}(\lambda) .
\end{aligned}
$$

Let us deal with $\Lambda_{n,+}$. We have that

$$
\begin{aligned}
\Lambda_{n,+}(\lambda) & =\frac{1}{n} \log \mathbb{E}\left(\exp \left(\lambda n \mathbf{Q}_{+, 1}^{n}\right)\right) \\
& =\frac{1}{n} \sum_{k=1}^{n} P\left(n \frac { \lambda } { 2 } \left(\sqrt{\int_{t_{k-1}^{n}}^{t_{k}^{n}} \sigma_{1, s}^{2} d s} \sqrt{\left.\left.\int_{t_{k-1}^{n}}^{t_{k}^{n}} \sigma_{2, s}^{2} d s+\int_{t_{k-1}^{n}}^{t_{k}^{n}} \sigma_{1, s} \sigma_{2, s} \rho_{s} d s\right)\right)}\right.\right. \\
& =\int_{0}^{1} P\left(\frac{\lambda}{2} f_{n}(t)\right) d t,
\end{aligned}
$$


where $P$ is given in $(2.3)$ and

$$
f_{n}(t):=\sum_{k=1}^{n} 1_{\left(t_{k-1}^{n}, t_{k}^{n}\right]}(t) \frac{\sqrt{\int_{t_{k-1}^{n}}^{t_{k}^{n}} \sigma_{1, s}^{2} d s} \sqrt{\int_{t_{k-1}^{n}}^{t_{k}^{n}} \sigma_{2, s}^{2} d s}+\int_{t_{k-1}^{n}}^{t_{k}^{n}} \sigma_{1, s} \sigma_{2, s} \rho_{s} d s}{t_{k}^{n}-t_{k-1}^{n}} .
$$

Let us remark that we have

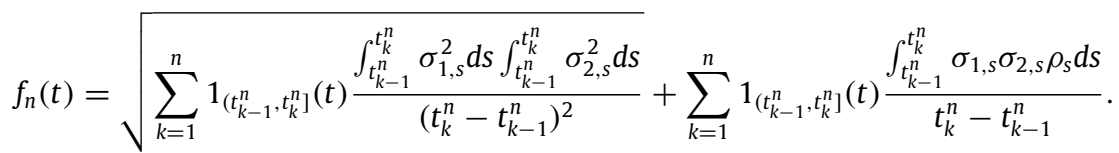

Clearly, $f_{n}(t)$ is a $d t$ martingale w.r.t. the partially directed filtration $\left(\mathscr{B}_{\tau_{n}}:=\sigma\left(\left(t_{k-1}^{n}, t_{k}^{n}\right], k=1, \ldots, n\right)\right)_{n}$. By the convexity of $P$ and Jensen inequality, we obtain that

$$
\int_{0}^{1} P\left(\frac{\lambda}{2} f_{n}(t)\right) d t \leq \int_{0}^{1} P\left(\frac{\lambda \sigma_{1, t} \sigma_{2, t}\left(1+\rho_{t}\right)}{2}\right) d t=\Lambda_{+}(\lambda) .
$$

On the other hand, by the classical Lebesgue derivation theorem, we have that

$$
f_{n}(t) \longrightarrow f(t):=\sigma_{1, t} \sigma_{2, t}+\sigma_{1, t} \sigma_{2, t} \rho_{t}, \quad d t-\text { a.e. on }[0,1] .
$$

The continuity of $P: \mathbb{R} \rightarrow(-\infty,+\infty]$ gives

$$
P\left(\frac{\lambda}{2} f_{n}(t)\right) \longrightarrow P\left(\frac{\lambda}{2} f(t)\right), \quad d t-\text { a.e. on }[0,1] .
$$

As $P\left(\frac{\lambda}{2} f_{n}(t)\right) \geq-\frac{|\lambda|}{2} \sigma_{1, t} \sigma_{2, t} \in L^{1}([0,1], d t)$, we can apply Fatou's lemma to conclude that

$$
\liminf _{n \rightarrow \infty} \Lambda_{n,+}(\lambda)=\liminf _{n \rightarrow \infty} \int_{0}^{1} P\left(\frac{\lambda}{2} f_{n}(t)\right) d t \geq \int_{0}^{1} \liminf _{n \rightarrow \infty} P\left(\frac{\lambda}{2} f_{n}(t)\right) d t=\Lambda_{+}(\lambda) .
$$

Doing the same calculations with $\Lambda_{n,-}$, we obtain that

$$
\Lambda_{n,-}(\lambda) \leq \int_{0}^{1} P\left(-\frac{\lambda \sigma_{1, t} \sigma_{2, t}\left(1-\rho_{t}\right)}{2}\right) d t=\Lambda_{-}(\lambda)
$$

and

$$
\liminf _{n \rightarrow \infty} \Lambda_{n,-}(\lambda) \geq \Lambda_{-}(\lambda) .
$$

From below, we conclude that

$$
\Lambda_{n}(\lambda) \leq \Lambda_{+}(\lambda)+\Lambda_{-}(\lambda):=\Lambda(\lambda),
$$

and

$$
\liminf _{n \rightarrow \infty} \Lambda_{n}(\lambda) \geq \Lambda(\lambda),
$$

which implies that

$$
\lim _{n \rightarrow \infty} \Lambda_{n}(\lambda)=\lim _{n \rightarrow \infty}\left(\Lambda_{n,+}(\lambda)+\Lambda_{n,-}(\lambda)\right)=\Lambda(\lambda),
$$

which ends the proof of first part of Proposition 2.1.

For the second part of Proposition 2.1, first we will reduce the study to the case $u_{l}=0$ for $l=1$, 2. Let $\beta=$ $\max \left(\left\|u_{1}\right\|_{\infty},\left\|u_{2}\right\|_{\infty}\right)$. Since

$$
\left|\mathbf{C}_{1}^{n}-\overline{\mathbf{C}}_{1}^{n}\right| \leq \frac{\beta^{2}}{n}+\frac{\beta}{n} \sum_{k=1}^{n}\left(\left|\int_{t_{k-1}^{n}}^{t_{k}^{n}} \sigma_{1, s} d B_{1, s}\right|+\left|\int_{t_{k-1}^{n}}^{t_{k}^{n}} \sigma_{2, s} d B_{2, s}\right|\right) .
$$

For $l=1,2$, we have for all $\lambda>0$ and all $\delta>0$

$$
\frac{1}{n} \log \mathbb{P}\left(\frac{1}{n} \sum_{k=1}^{n}\left|\int_{t_{k-1}^{n}}^{t_{k}^{n}} \sigma_{l, s} d B_{l, s}\right| \geq \delta\right) \leq-\delta \lambda+\frac{\lambda^{2}}{2 n} \int_{0}^{1} \sigma_{l, s}^{2} d s .
$$


Letting $n$ go to infinity and then $\lambda$ to infinity we get that for all $\delta>0$

$$
\lim _{n \rightarrow \infty} \frac{1}{n} \log \mathbb{P}\left(\frac{1}{n} \sum_{k=1}^{n}\left|\int_{t_{k-1}^{n}}^{t_{k}^{n}} \sigma_{l, s} d B_{l, s}\right| \geq \delta\right)=-\infty .
$$

By the approximation technique (Theorem 4.2.13 in Dembo and Zeitouni (1998)), we deduce that $\mathbb{P}\left(\mathbf{C}_{1}^{n} \in \cdot\right)$ satisfies the same LDP as $\mathbb{P}\left(\overline{\mathbf{C}}_{1}^{n} \in \cdot\right)$. Hence we can assume that $u_{l}=0$ for $l=1,2$.

Now, by inspection of the proof of Theorem 1.1 in Djellout et al. (1999), we deduce that the sequence $\mathbb{P}\left(\mathbf{Q}_{ \pm, 1}^{n} \in \cdot\right)$ satisfies the LDP on $\mathbb{R}$ with speed $n$ and rate function given by

$$
\Lambda_{ \pm}^{*}(x)=\sup _{\lambda \in \mathbb{R}}\left\{\lambda x-\Lambda_{ \pm}(\lambda)\right\}
$$

By the independence of the sequences $\mathbf{Q}_{+, 1}^{n}$ and $\mathbf{Q}^{n}{ }_{, 1}$, and the contraction principle, see Exercise 4.2.7 in Dembo and Zeitouni (1998), we deduce that $\mathbb{P}\left(\overline{\mathbf{C}}_{1}^{n} \in \cdot\right)$ satisfies the LDP with rate function

$$
\Lambda^{*}(x)=\inf _{x=x_{1}-x_{2}}\left\{\Lambda_{+}^{*}\left(x_{1}\right)+\Lambda_{-}^{*}\left(x_{2}\right)\right\} .
$$

As we have also determined explicitly the logarithm of the moment generating function $\Lambda$, the rate function is also given by (2.1).

Proof of Theorem 2.2. The proof of the first part is very similar to Proposition 2.1. It is a consequence of Theorem 1.2 in Djellout et al. (1999) and the contraction principle. For the second part of Theorem 2.2, the same arguments give the large deviation with the rate function

$$
I(\gamma)=\inf _{\gamma=\gamma_{1}-\gamma_{2}}\left\{I_{+}\left(\gamma_{1}\right)+I_{-}\left(\gamma_{2}\right)\right\}
$$

where

$$
I_{ \pm}(\gamma)=\int_{0}^{1} P^{*}\left(\frac{2 \dot{\gamma}(t)}{\sigma_{1, t} \sigma_{2, t}\left(1 \pm \rho_{t}\right)}\right) d t+\int_{0}^{1} \frac{1}{\sigma_{1, t} \sigma_{2, t}\left(1 \pm \rho_{t}\right)} d \gamma^{\perp} .
$$

An easy variational calculus gives the identification of the rate function in (2.4).

Proof of Theorem 2.3. As before, we treat only the case $u_{l}=0$ for $l=1,2$. We have the following decomposition:

$$
\mathbf{C}^{n}-\left\langle X_{1}, X_{2}\right\rangle=\left(\mathbf{Q}_{+, \cdot}^{n}-\alpha_{+, .}\right)-\left(\mathbf{Q}_{,,}^{n}-\alpha_{-, .}\right),
$$

where the definition of $\alpha_{ \pm}$is given in (2.9).

Now using Theorem 1.3 in Djellout et al. (1999), we deduce that $\mathbb{P}\left(\frac{\sqrt{n}}{b_{n}}\left(\mathbf{Q}_{ \pm, \cdot}^{n}-\alpha_{ \pm, .}\right) \in \cdot\right)$ satisfies the LDP on $\mathbb{P}_{0}([0,1])$ with speed $b_{n}^{2}$ and with the good rate function $J_{ \pm, m}$ given by

$$
J_{ \pm, m}(\gamma)=\left\{\begin{array}{l}
\int_{0}^{1} \frac{\dot{\gamma}(t)^{2}}{\sigma_{1, t}^{2} \sigma_{2, t}^{2}\left(1 \pm \rho_{t}\right)^{2}} 1_{\left[t: \sigma_{1, t} \sigma_{2, t}>0\right]} d t \quad \text { if } d \gamma \ll \sigma_{1, t} \sigma_{2, t}\left(1 \pm \rho_{t}\right) d t \\
+\infty \text { otherwise. }
\end{array}\right.
$$

By the same argument as before, we deduce that $\mathbb{P}\left(\frac{\sqrt{n}}{b_{n}}\left(\mathbf{C}^{n}-\left\langle X_{1}, X_{2}\right\rangle.\right) \in \cdot\right)$ satisfies the LDP on $\mathbb{D}_{0}([0,1])$ with speed $b_{n}^{2}$ and with the good rate function $J_{m}$ given by

$$
J_{m}(\gamma)=\inf _{\gamma=\gamma_{1}-\gamma_{2}}\left\{J_{+, m}\left(\gamma_{1}\right)+J_{-, m}\left(\gamma_{2}\right)\right\} .
$$

An easy calculation gives the identification of the rate function in (2.6).

\section{Acknowledgments}

The authors want to thank Professor Liming Wu for fruitful discussions. We are grateful to the anonymous referee for the valuable comments.

\section{References}

Ait-Sahalia, Y., Fan, J., Xiu, D., 2010. High-frequency covariance estimates with noisy and asynchronous financial data. J. Amer. Statist. Assoc. 105 (492), $1504-1517$.

Dalalyan, A., Yoshida, N., 2011. Second-order asymptotic expansion for a non-synchronous covariation estimator. Ann. Inst. Henri Poincaré Probab. Stat. 47 (3), 748-789. 
Dembo, A., Zeitouni, O., 1998. Large Deviations Techniques and Their Applications, 2nd ed.. In: Applications of Mathematics, vol. 38. Springer-Verlag, New York.

Djellout, H., Guillin, A., Wu, L., 1999. Large and moderate deviations for estimators of quadratic variational processes of diffusions. Stat. Inference Stoch. Process. 2, 195-225.

Hayashi, T., Yoshida, N., 2011. Nonsynchronous covariation process and limit theorems. Stochastic Process. Appl. 121, 2416-2454.

Jiang, H., 2010. Moderate deviations for estimators of quadratic variational process of diffusion with compound Poisson jumps. Statist. Probab. Lett. 80, $1297-1305$.

Kanaya, S., Otsu, T. 2012. Large deviations of realized volatility. Stochastic Process. Appl. 122, 546-581.

Mancini, C., 2008. Large deviation principle for an estimator of the diffusion coefficient in a jump-diffusion process. Statist. Probab. Lett. 78, 869-879.

Mancini, C., Gobbi, F., 2012. Identifying the brownian covariation from the co-jumps given discrete observations. Econometric Theory 28 (2), $249-273$. 